\title{
Long-range electrothermal fluid motion in microfluidic
}

\section{systems}

Yi Lu๋, Qinlong Ren+, Tingting Liu ${ }^{\dagger}$, Siu Ling Leung ${ }^{\ddagger}$, Vincent Gaull, Joseph C. Liao ${ }^{\perp}$, Cho Lik Chan ${ }^{\dagger}$ and Pak Kin Wongt,

† Department of Aerospace and Mechanical Engineering, The University of Arizona, Tucson, Arizona, 85721, USA.

‡ College of Medicine, The University of Arizona, Tucson, 85724, USA

|| GeneFluidics Inc., Irwindale, California, 91010, USA

$\perp$ Department of Urology, Stanford University, Stanford, California, 94305, USA

${ }^{*}$ Correspondence should be addressed to Dr. Pak Kin Wong. Tel: +1-520-626-2215; Fax: +1 520-621-8191; E-mail: pak@email.arizona.edu 


\section{Abstract}

AC electrothermal flow (ACEF) is the fluid motion created as a result of Joule heating induced temperature gradients. ACEF is capable of performing major microfluidic operations, such as pumping, mixing, concentration, separation and assay enhancement, and is effective in biological samples with a wide range of electrical conductivity. Here, we report long-range fluid motion induced by ACEF, which creates centimeter-scale vortices. The long-range fluid motion displays a strong voltage dependence and is suppressed in microchannels with a characteristic length below $\sim 300 \mu \mathrm{m}$. An extended computational model of ACEF, which considers the effects of the density gradient and temperature-dependent parameters, is developed and compared experimentally by particle image velocimetry. The model captures the essence of ACEF in a wide range of channel dimensions and operating conditions. The combined experimental and computational study reveals the essential roles of buoyancy, temperature rise, and associated changes in material properties in the formation of the longrange fluid motion. Our results provide critical information for the design and modeling of ACEF based microfluidic systems toward various bioanalytical applications.

Keywords: AC electrothermal flow, electrokinetics, microfluidics, buoyancy, computational fluid dynamics 


\title{
Nomenclature
}

\author{
C specific heat capacity of the fluid \\ E magnitude of the electric field \\ Erms root mean square electric field \\ $f$ frequency of the AC voltage \\ $f_{c} \quad$ crossover frequency \\ $F_{E} \quad$ time-averaged electrothermal force \\ $g$ gravitational acceleration \\ I identity matrix \\ $J$ the current density \\ $k$ thermal conductivity \\ $L \quad$ characteristic length \\ $\hat{n}_{\theta} \quad$ unit vector in tangential direction \\ $p$ pressure \\ $P e \quad$ Péclet number \\ $\mathrm{Ra} \quad$ Rayleigh number \\ $T$ temperature \\ $u \quad$ fluid velocity \\ $V_{p p} \quad$ peak to peak voltage \\ $V_{r m s}$ root mean square voltage
}

\section{Greek symbols}

$\alpha \quad$ parameter defined as $\alpha=(\partial \varepsilon / \partial T) / \varepsilon$

$\beta \quad$ parameter defined as $\beta=(\partial \sigma / \partial T) / \sigma$

$\varepsilon \quad$ electrical permittivity

$\mu \quad$ viscosity of the solution

$\rho_{m} \quad$ medium density

$\rho_{q} \quad$ charge density

$\sigma \quad$ electrical conductivity

$\tau \quad$ ratio of $\varepsilon(T) / \sigma(T)$

$\phi \quad$ electrical potential

$\omega \quad$ angular frequency of the $\mathrm{AC}$ voltage, $\omega=2 \pi f$ 


\section{Introduction}

AC electrokinetics is a promising technology for the development of integrated point-ofcare diagnostic systems [1, 2]. AC electrokinetic phenomena, such as AC electrothermal flow (ACEF), enables fluid manipulation for automating bioanalytical procedures. ACEF arises from Joule heating induced temperature gradients developed within a fluid when an external electric field is applied [3]. The temperature gradients create local electrical conductivity, permittivity, viscosity, and density gradients in the solution. These gradients and their interactions with the electric field, in turn, create bulk fluid force and motion. ACEF is effective for fluids with a large range of electrical conductivity and is particularly useful for manipulating conductive physiological samples and biological buffers. By properly designing the electrokinetic devices, ACEF has been demonstrated for pumping, mixing, concentration, separation, signal enhancement and noise reduction [4-18].

A theoretical model of ACEF was developed for parallel electrodes with a small gap [3]. In this model, the equilibrium temperature distribution was determined by considering the energy balance equation. The effect of fluid motion on the temperature distribution was ignored due to the low velocity. The gradients in permittivity and electrical conductivity, which interact with the electric field to generate the bulk fluid force, were approximated based on their linear temperature sensitivity. The buoyancy effect was not considered due to the small length scale of microfluidics. This classical model forms the basis of numerous ACEF studies and facilitates the design of various bioanalytical devices. With the rapidly expanding applications of ACEF, the classical model, however, does not fully describe the diverse behaviors of ACEF observed in microfluidic systems. Discrepancies between the fluid motion and the classical model were reported at high voltages and conductivities $[9,19,20]$. Several modifications of 
the classical model, including buoyancy effects, temperature-dependent parameters and convective heat transfer, have been proposed. Nevertheless, the effects of these modifications and the diverse behaviors of ACEF are only partially understood. A systematic experimental investigation along with modeling and simulation is required to decipher the diverse behaviors of ACEF and provide guidelines on the design of ACEF based bioanalytical devices.

In this study, we investigate an uncharacterized long-range fluid motion induced by ACEF, which creates centimeter scale vortices. The long-range fluid motion is studied under various channel dimensions, sample properties, and operating parameters. Microfluidic channels with characteristic lengths from $100 \mu \mathrm{m}$ to $1 \mathrm{~mm}$ are designed to investigate the temperature and velocity profiles. Fig. 1a-b shows the cross-sectional and top views of the microchannel. The fluid motion and the temperature profile in the microchannel are experimentally characterized by particle image velocity and infrared thermometry. The effects of sample electrical conductivity, voltage, and frequency, are investigated systematically. An extended computational model of ACEF, which describes the effects of the density gradient and temperature-dependent parameters, is also developed to compare with the experimental results. Using the combined experiment and computation approach, we elucidate the effects of buoyancy, convective heat transfer, boundary conditions, and temperature-dependent parameters on the long-range electrothermal fluid motion. 

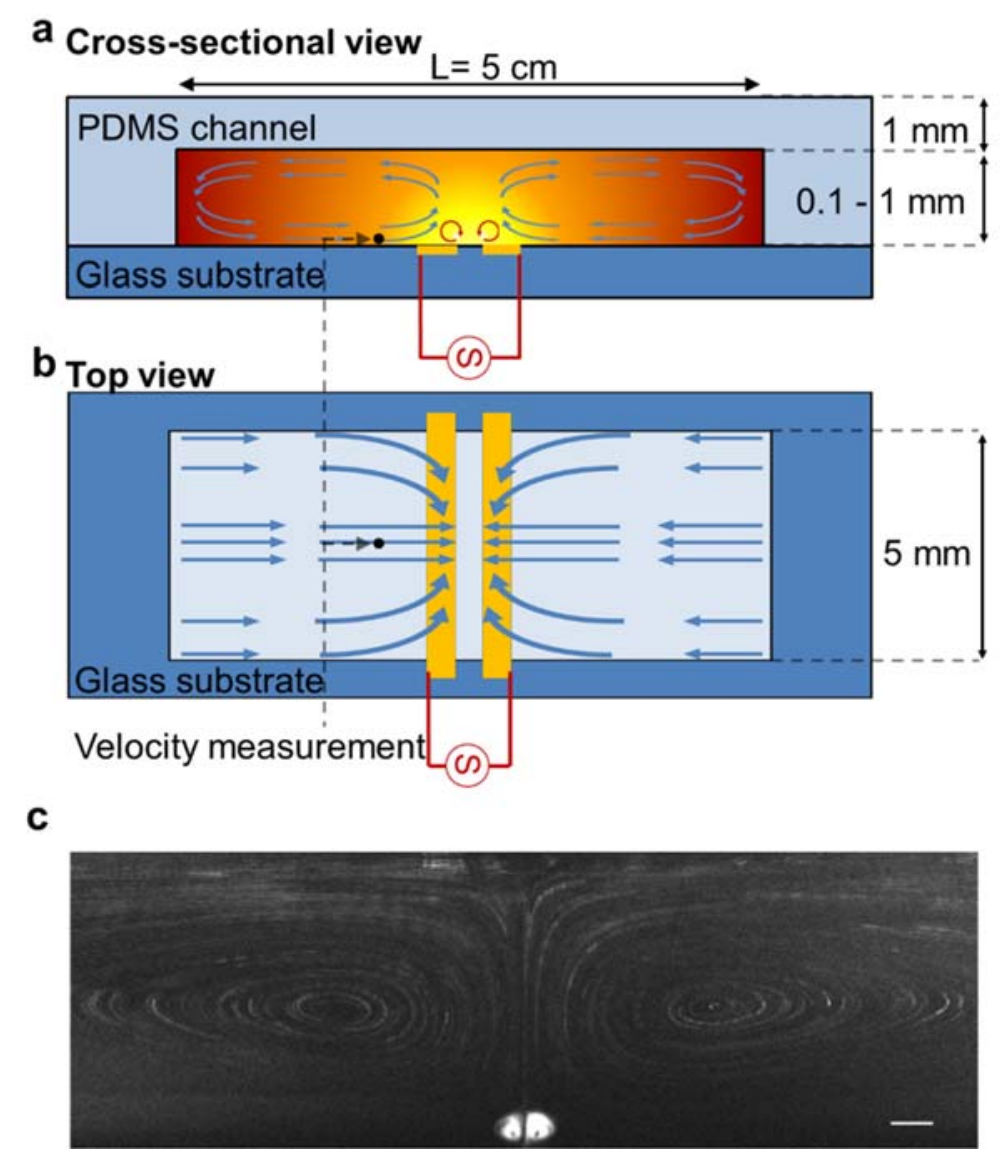

Figure 1. Long-range fluid motion induced by ACEF. (a-b) Schematic of the (a) cross sectional view and (b) top view of the microchannel integrated with a pair of parallel electrodes. Arrows indicate the direction of the flow. Black spots represent the location of velocity measurement. (c) Formation of two pairs of counter vortices in the microchannel. Fluorescence images were stacked together to illustrate the trajectories of the microspheres. Scale bar, $100 \mu \mathrm{m}$.

\section{MATERIALS AND METHODS}

\section{Microfabrication}


The microfluidic device consisted of a pair of parallel electrodes in a polydimethylsiloxane (PDMS) microchannel (supplementary Fig. S1). The length, width and distance of the electrodes were $7 \mathrm{~mm}, 100 \mu \mathrm{m}$, and $50 \mu \mathrm{m}$ respectively. The electrodes were fabricated by evaporation and lift-off on glass microscope slides (Thermo scientific, Portsmouth, NH). Briefly, the glass slides were cleaned by acetone and isoamyl alcohol, dried with nitrogen, and baked for $15 \mathrm{~min}$ at $125{ }^{\circ} \mathrm{C}$. The electrode pattern was defined by photolithography with AZ-3312 photoresist (AZ Electronic Material, Branchburg, NJ). The electrodes were deposited by evaporating $50 \mathrm{~nm}$ of titanium, $150 \mathrm{~nm}$ of gold, and $50 \mathrm{~nm}$ of titanium onto the glass slides followed by the lift-off process with acetone. The microchannels were fabricated by PDMS molding. The mold was created by a rapid prototyping process based on a computer numerical control system with micrometer precision (HAAS automation Inc., Oxnard, CA). The length and width of the microchannel were $5 \mathrm{~cm}$ and $5 \mathrm{~mm}$ respectively. The depth of the microchannel varied from $100 \mu \mathrm{m}$ to $1000 \mu \mathrm{m}$ for the theoretical and experimental study of ACEF. PDMS was mixed at 1:10 curing agent to elastomer base ratio and incubated at $65^{\circ} \mathrm{C}$ for 5 hours. Inlets and outlets were punched into the PDMS chamber by a $0.7 \mathrm{~mm}$ Harris Uni-Core punch tool (Ted Pella, Inc., Redding, CA). The sample

chamber and electrode glass substrate were sterilized and bonded by air plasma treatment (Harrick Plasma, Ithaca, NY). An assembled device is shown in supplementary Fig. S1.

\section{Experimental setup}

The microfluidic chip was loaded on a Leica DMI-4000B inverted epi-fluorescence microscope (Leica Microsystems, Wetzlar, Germany). A Nikon Eclipse E800 upright epifluorescence microscope (Nikon Instruments INC., Melville, NY) was also used to observe the 
fluid motion on the electrode. Images and videos were captured using a Cooke SensiCAM QE CCD camera (PCO-TECH Inc., Romulus, MI). A 5 mm prism (PS609, Thorlabs, Newton, NJ) was setup on the inverted microscope to monitor the fluid motion from the side (supplementary Fig. S2). A function generator (33220A, Agilent, Englewood, CO) was used for generating the AC potential. The AC potential was monitored by a digital oscilloscope (DSOX2012A, Agilent, Englewood, CO) during the experiment.

\section{Image processing and particle image velocimetry}

Fluorescent microspheres (F8810; Invitrogen, Carlsbad, CA) with a diameter of $200 \mathrm{~nm}$ were applied for particle image velocimetry. The particles were diluted using Mueller Hinton $(\mathrm{MH})$ broth with an electrical conductivity $\sim 1.1 \mathrm{~S} / \mathrm{m}\left(\right.$ at $\left.21^{\circ} \mathrm{C}\right)$. The images and videos were taken by a $10 x$ objective (Leica HC PL FL, NA $=0.3$ ) and processed using the NIH ImageJ software. The velocity field was calculated using the open source JPIV software package. The depth of focus for PIV measurement was approximately $8 \mu \mathrm{m}$. The CCD pixel size was $6.4 \mu \mathrm{m}$. The measurement uncertainty can be calculated applying the following formula $\delta_{x} \approx$ $\frac{d_{e}}{10 \mathrm{M}}=20 \mathrm{~nm}$, where $d_{\mathrm{e}}$ is the effective particle diameter $[21,22]$. In the experiment, the particle density was 6 - 8 particles in each $128 \times 128$ pixel interrogation window. All velocity measurements were performed $100 \mu \mathrm{m}$ above the bottom of the channel surface and $500 \mu \mathrm{m}$ away from the electrode (Fig. 1a-b). The setting was optimized for electrohydrodynamic measurement with minimal influence of dielectrophoresis [23, 24].

\section{Temperature measurement}


The temperature profiles were measured by infrared thermometry and calibrated by fine gage thermocouples. An infrared camera system SC6700 (FLIR, Boston, MA) was applied for measuring the temperature distribution inside the microchannel. The infrared camera measured the mid-wavelength infrared (MWIR), covering $3 \mu \mathrm{m}$ to $5 \mu \mathrm{m}$. For a $1 \mathrm{~mm}$ thick soda-lime-silica glass, the transmittance is approximately $\sim 70 \%$ for $2.5 \mu \mathrm{m}$ to $4 \mu \mathrm{m}$ wavelength and the value drops to $\sim 0 \%$ for $5 \mu \mathrm{m}$ or larger wavelength. Unlike long-wavelength infrared cameras that detect only the surface temperature, the MWIR camera measures the temperature inside the microchannel. The temperature measurement was calibrated using fine gage thermocouples (Omega Engineering, INC., Stamford, Connecticut). The thermocouples, which can be as small as $13 \mu \mathrm{m}$, were embedded in the PDMS microchannel. The small size of the thermocouple allowed a good spatial resolution with a small loading effect for the measurement.

\section{Computational simulation}

The computational model of ACEF was implemented in COMSOL Multiphysics v4.4 (COMSOL Inc., Los Angeles, CA). A 2D simulation including the PDMS chamber and glass substrate was implemented (Fig. 1a-b). The entire domain was mashed by free triangular method with total number of 53,087 elements (supplementary Fig. S3). The convectiondiffusion and heat transfer modules were applied for calculating the electric field and temperature distributions in the model. The velocity field was determined using the laminar flow module. The two body forces, electrothermal force and buoyancy force, were incorporated in the calculation. The temperature-dependent physical properties used in the 
calculation are listed in Table 1. Finally, the velocity and temperature results of the whole domain can be plotted as supplementary Fig. S4.

\section{RESULTS}

\section{Long-range fluid motion induced by ACEF}

The fluid motion was monitored at the top and bottom planes of the microchannel (supplementary Video S1-2). A prism was also used to directly measure the fluid motion from the cross section (supplementary Video S3-5). Fig. 1c shows stacking of fluorescence images to illustrate the trajectories of the microspheres. In agreement with previous ACEF studies [3], a pair of counter vortices driving from the gap to the electrode surface was observed near the electrode. Interestingly, a long-range fluid motion moving from the channel surface to the gap forming two centimeter-scale counter vortices was also observed in the microchannel. To characterize this long-range ACEF induced fluid motion, the voltage and frequency were studied systematically (Fig. 2). The experiments were performed using samples with conductivities from 0.6 to $2.4 \mathrm{~S} / \mathrm{m}$, which covers the electrical conductivity of most physiological fluids and biological buffers. In our experiment, the velocity of the fluid motion increased with the applied voltage and electrical conductivity (Fig. 2a). In contrast, the velocity showed weak dependence with the applied frequency (Fig. 2b). The velocity profiles in the horizontal and vertical directions are shown in Fig. 2c-d, confirming the formation of the large counter vortices in the microchannel. The classical model predicts ACEF increases linearly with the electrical conductivity and to the fourth power of the voltage, i.e., $u \sim \sigma V^{4}$. The data, however, did not fit into the power function and the discrepancy was particularly significant at high voltage and electrical conductivity values. 

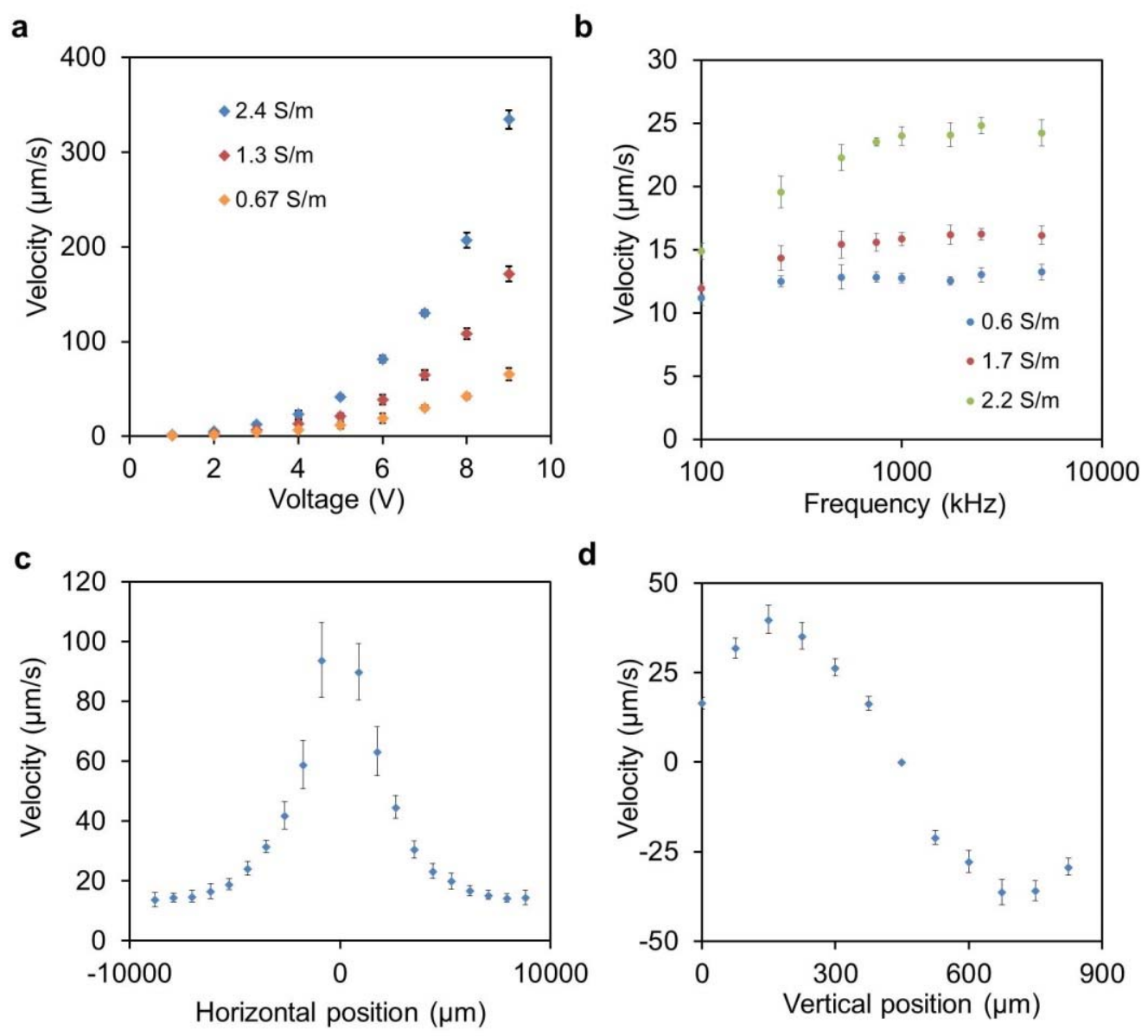

Figure 2. Characterization of the long-range fluid motion. (a) The voltage dependence of the fluid velocity. (b) The frequency dependence of the fluid velocity for different conductivities. The experiments were performed in samples with different conductivities. (c-d) Fluid velocity profiles of the horizontal and vertical directions. Experiments were performed using $800 \mu \mathrm{m}$ height channels.

\section{Extended computational model of ACEF}

To investigate the discrepancy, we developed an extended computational model of ACEF by modifying the theoretical model developed by Ramos et al. [3]. The mathematical formulation involves a set of coupled equations describing the electric field, energy balance, 
and fluid motion [25]. The electrical problem considers the Gauss's law, charge conservation, and Faraday's Law (equations 1-3).

$$
\begin{gathered}
\nabla \cdot(\varepsilon \mathrm{E})=\rho_{q} \\
\frac{\partial \rho_{q}}{\partial t}+\nabla \cdot J=0 \\
\nabla \times E=0
\end{gathered}
$$

where $\varepsilon$ is the solution permittivity, $\rho_{q}$ is the local charge density, and $J$ is the current density. The current density is defined by the Ohm's law: $J=\sigma E$, where $\sigma$ is the electrical conductivity of the solution and $E$ is the electric field. By using perturbation analysis, the electrical potential $\phi$ in the medium is estimated by equation 4 .

$$
\nabla^{2} \phi=0, E=-\nabla \phi
$$

When an electrical field is applied, Joule heating is induced in the solution. The heat generation per unit volume and the energy equation for determining the temperature distribution are given by equations 5-6.

$$
\begin{gathered}
W=\sigma \cdot E^{2} \\
\rho_{m} c\left(\frac{\partial T}{\partial t}+u \cdot \nabla T\right)=k \nabla^{2} T+\sigma E^{2}
\end{gathered}
$$

where $\rho_{m}, k$, and $c$ are the density, thermal conductivity, and specific heat of the fluid, respectively. Once the electric field and the temperature field are determined, the time averaged electrothermal force can also be estimated. A general expression for the electrostatic force on a bulk fluid is given by equation 7 .

$$
F_{e}=\rho_{q} E-\frac{1}{2} E^{2} \nabla \varepsilon+\frac{1}{2} \nabla\left(\rho_{m}\left(\frac{\partial \varepsilon}{\partial \rho_{m}}\right) E^{2}\right)
$$


The first and second terms of equation 7 describe the Coulomb force and the dielectric force. The third term represents electrostriction, which is omitted for incompressible fluid. The time-averaged force per unit volume is given by equation 8 .

$$
\mathrm{F}_{\text {electrothermal }}=\frac{1}{2} \cdot \frac{\varepsilon(\alpha-\beta)}{1+(\omega \varepsilon / \sigma)^{2}}(\nabla T \cdot E) E-\frac{1}{4} \varepsilon \alpha|E|^{2}
$$

where $\omega=2 \pi f$ is the angular frequency of the AC potential, the gradients in permittivity and electrical conductivity can be present as $\alpha=(\partial \varepsilon / \partial T) / \varepsilon$ and $\beta=(\partial \sigma / \partial T) / \sigma$ respectively. The model predicts a transition of the dominance between the Coulomb force and the dielectric force when $\omega \varepsilon / \sigma$ is approximately 1 .

A temperature gradient also gives rise to a change in fluid density. In the extended model, the effects of density gradient and buoyancy force is considered. The buoyancy force is described by equation 9 .

$$
\mathrm{F}_{\text {buoyancy }}=g \cdot\left(\rho-\rho_{r}\right)
$$

The velocity vector field, $u$, of the system is estimated by incorporating the two body forces to the fluid mechanics equations. The velocity field is modeled by the continuity and Navier-Stokes equations at low Reynolds number (equations 10-11).

$$
\begin{gathered}
\nabla \cdot u=0 \\
0=-\nabla p+\mu \nabla^{2} u+F_{\text {electrothermal }}+F_{\text {buoyancy }}
\end{gathered}
$$

where $p$ is the pressure and $\mu$ the viscosity of the fluid.

For samples with a low electrical conductivity, the temperature rise is typically small. For instance, applying an AC potential of $20 \mathrm{Vpp}$ to a $0.01 \mathrm{~S} / \mathrm{m}$ solution results in a temperature rise of only $\sim 1^{\circ} \mathrm{C}$. However, the temperature can be significantly higher for samples with a high electrical conductivity. When an $\mathrm{AC}$ potential of $10 \mathrm{Vpp}$ is applied to a 0.1 $\mathrm{M} \mathrm{KCl}$ solution $\left(\sigma=1.16 \mathrm{~S} / \mathrm{m}\right.$ at $\left.20^{\circ} \mathrm{C}\right)$, the temperature rise can reach over $20^{\circ} \mathrm{C}$. For such a 
large temperature rise, the electrical conductivity itself increases for $\sim 40 \%\left(\sigma=1.66\right.$ at $\left.40^{\circ} \mathrm{C}\right)$, which in turn significantly enhances the Joule heating effect. Therefore, the temperature dependent properties, such as permittivity, thermal conductivity, dynamic viscosity, density and specific heat capacity, are considered. Therefore, the equations are modified to incorporate the temperature-dependent parameters. The heat generation in equation 5 and the conservation of energy in equation 6 are modified to:

$$
\begin{gathered}
W=\sigma(T) \cdot E^{2} \\
\rho_{m}(T) c(T)\left(\frac{\partial T}{\partial t}+\boldsymbol{u} \cdot \nabla T\right)=\nabla \cdot(k(T) \nabla T)+\sigma(T)(\boldsymbol{E} \cdot \boldsymbol{E})
\end{gathered}
$$

Furthermore, the classical model of the ACEF assumes that the permittivity and electrical conductivity changes with temperature are small. For a small temperature rise, the temperature sensitivities of permittivity and electrical conductivity can be estimated by $\varepsilon(T)=$ $\varepsilon^{0}\left(1+\alpha\left(T-T_{0}\right)\right) ; \sigma(T)=\sigma^{0}\left(1+\beta\left(T-T_{0}\right)\right)$, where $\alpha \approx-0.0046 \mathrm{~K}^{-1}, \beta \approx 0.020 \mathrm{~K}^{-1}$ at $20^{\circ} \mathrm{C}[3$, 25]. In the extended model, the time-averaged electrothermal force, continuity and the NavierStokes equations are modified to include temperature dependent parameters (equations 1416).

$$
\begin{gathered}
F_{\text {electrothermal }}=\frac{1}{2} \cdot \frac{\left(\frac{\partial \varepsilon}{\partial T}-\tau \frac{\partial \sigma}{\partial T}\right)}{1+(2 \pi f \tau)^{2}}(\nabla T \cdot E) E-\frac{1}{4}(\partial \varepsilon / \partial T)|E|^{2} \nabla T \\
\nabla \cdot(\rho u)=0 \\
0=\nabla \cdot\left[-p I+\mu\left(\nabla u+(\nabla u)^{T}\right)-\frac{2}{3} \mu(\nabla \cdot u) I\right]+\boldsymbol{F}
\end{gathered}
$$

where $\tau=\varepsilon(T) / \sigma(T), I$ is identity matrix. 
In existing models of ACEF, the boundary conditions are often considered to be the ambient temperature. The fixed boundary condition, however, does not represent the large temperature rise observed in our experiment. To determine the appropriate boundary condition, the surface temperature of the PDMS chip was measured by infrared thermometry (supplementary Fig. S5). A high temperature was observed on the surface of PDMS. To reflect this observation, the third type boundary conditions (convective heat flux) were applied to the PDMS and glass surfaces in the simulation (equation 17).

$$
-k \frac{\partial T}{\partial y}=h\left(T-T_{a i r}\right)
$$

\section{Temperature profiles in the microchannel}

To evaluate the extended computational model, we computed the temperature profiles and compared with the experimental data using an $800 \mu \mathrm{m}$ height channel. The temperature distributions with $7 \mathrm{~V}$ peak to peak were calculated in three different scenarios: the classical model, the classical model with temperature dependent parameters $(\mathrm{w} / \mathrm{T})$ and the extended model with temperature dependent parameters and the third type boundary condition $(\mathrm{w} / \mathrm{T}+$ w/B.C.). Compared to the classical model, the extended model significantly expanded the temperature gradient and increased the temperature rise (Fig. 3a). Fig. 3b shows the temperature profile measured by infrared thermometry under the same condition. The extended model matched perfectly with the experimental data $\left(R^{2}>0.99\right)$. The voltage dependence of the temperature rise was also compared quantitatively in different scenarios

(Fig. 3c). For small voltages (e.g., small temperature rise), all three models were in reasonable agreement with the experimental data. For a large voltage, a large discrepancy 
was observed in the classical model and classical model with temperature dependent parameters. In contrast, the extended model of ACEF captured the voltage dependence on the temperature rise. The data demonstrate the importance of the boundary condition and support the applicability of the extended model for studying ACEF.

a
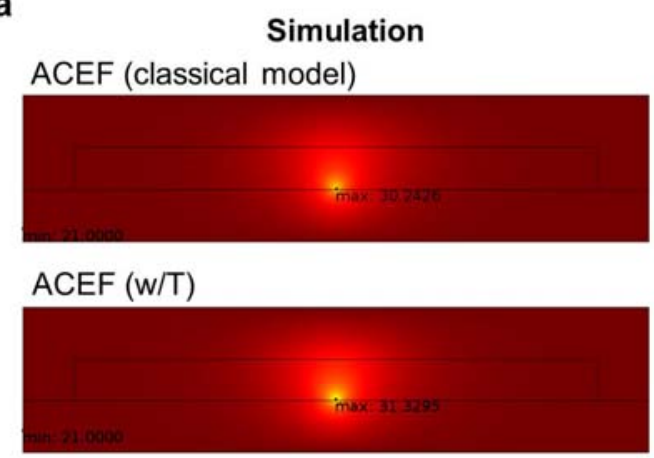

ACEF (w/T + w/B.C.)

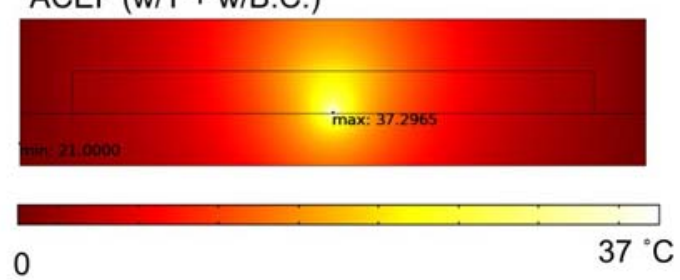

b

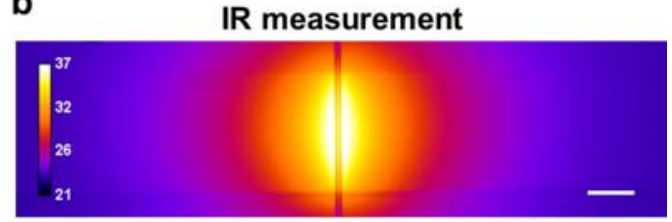

c

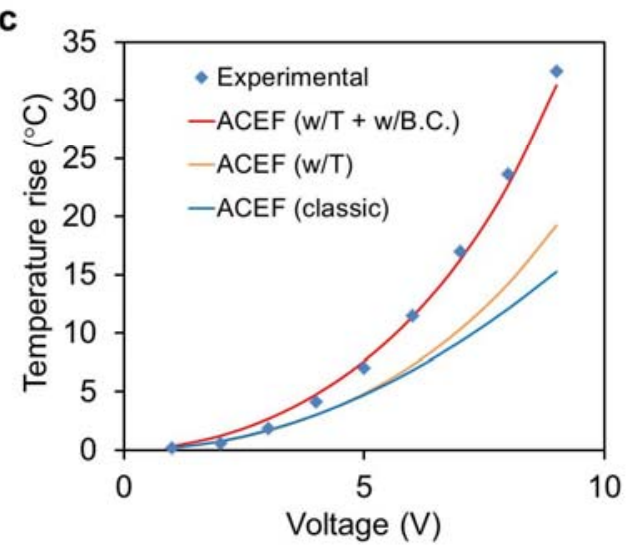

Figure 3. Temperature profiles in the microchannel. (a) The temperature profile calculated using the classical model of ACEF (classic), classical model with temperature dependent parameters (w/T), and the extended model with temperature parameters and third type boundary condition (w/T+W/B.C.). (b) The temperature profile measured by infrared thermometry near the bottom of the channel. Scale bar, $2 \mathrm{~mm}$. (c) Voltage dependence of the temperature rise determined experimentally and computationally. The coefficient of determination of different model were calculated respectively, $R^{2}=0.995754$ (for ACEF w/T + w/B.C.), $R^{2}=0.676707$ (for ACEF $w / T$ ) and $R^{2}=0.495894$ (for ACEF classic). 
In the classical model of ACEF, the length scale of the microchannel is assumed to be small. For practical applications, the characteristic length of the microchannel can often be over $100 \mu \mathrm{m}$ which may affect the significance of heat convection and buoyancy. We investigated the effects of the characteristic length by adjusting the channel height in the extended model. Fig. 4 shows the velocity fields in channels with four different heights: 100 , 200,300 , and $800 \mu \mathrm{m}$. Only the small counter vortices near the electrodes were observed in small channels (e.g., $100 \mu \mathrm{m}$ and $200 \mu \mathrm{m}$ ). The small vortices showed weak dependence on the channel dimension. Long-range fluid motion and large vortices were observed in larger channels. The long-range fluid motion was observed only for channels with a height of $300 \mu \mathrm{m}$ or higher. In other words, a transition length scale between $200 \mu \mathrm{m}$ and $300 \mu \mathrm{m}$ exists for the long-range fluid motion. The size of the vortices and velocity generally increased with the height of the channel. Remarkably, the same effect of the characteristic length was observed in the experiments. Fig. 5 compares the velocity profiles from computational simulation and particle image velocimetry in a $300 \mu \mathrm{m}$ height channel. Fig. 5a shows the velocity profile in the cross-sectional plane calculated by the extended model. Fig. 5b shows the PIV measurement of the velocity at the bottom layer and top layer in the channel. The computational model matched the velocity profile quantitatively. In general, the extended model was capable of predicting the long-range fluid motion observed in the experiment and was applied for studying the factors controlling the centimeter-scale vortices. 

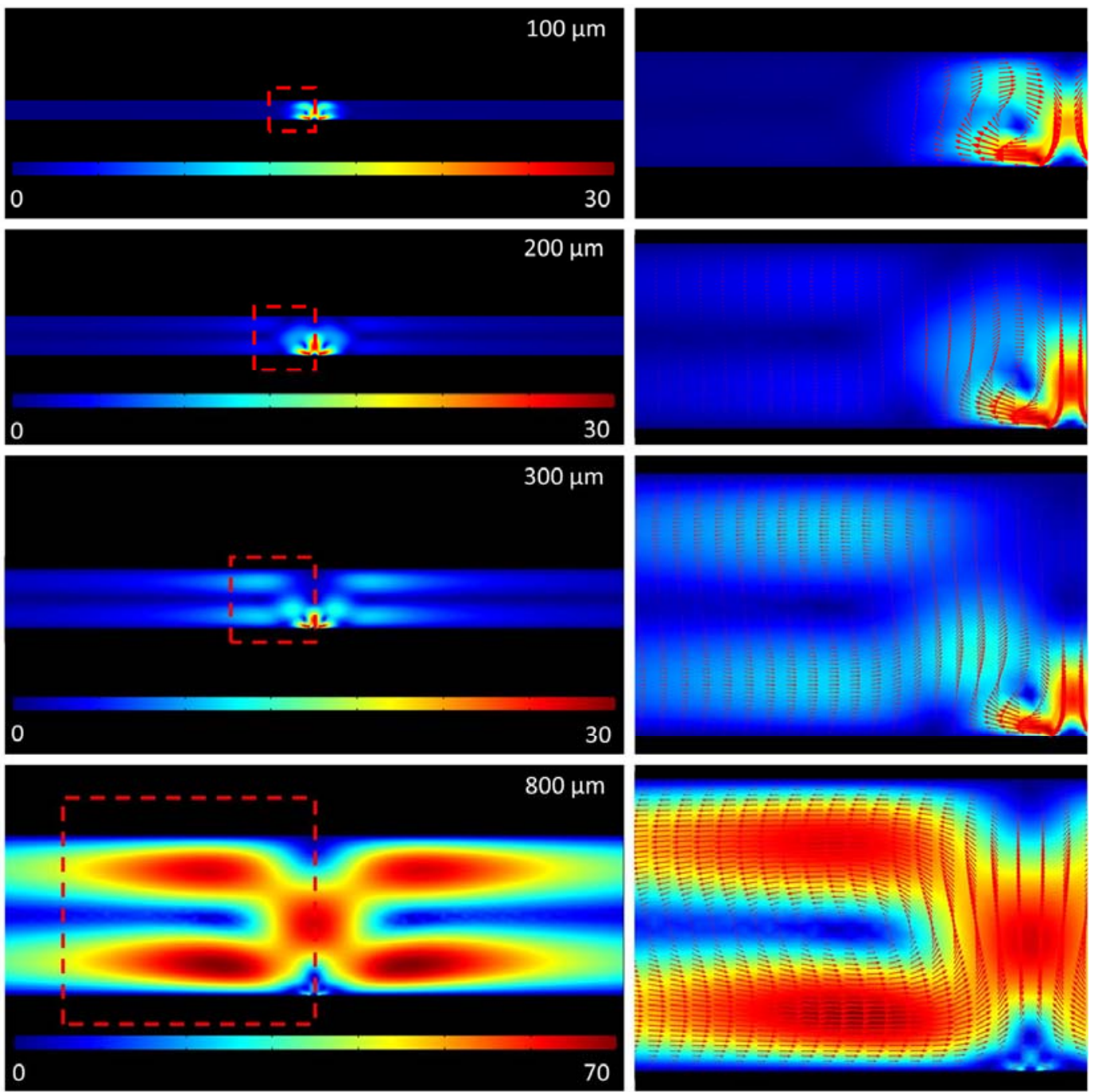

Figure 4. The velocity profiles in channels with different heights: $100 \mu \mathrm{m}, 200 \mu \mathrm{m}, 300 \mu \mathrm{m}$ and $800 \mu \mathrm{m}$. Unit: $\mu \mathrm{m} / \mathrm{s}$. 
a

Simulation

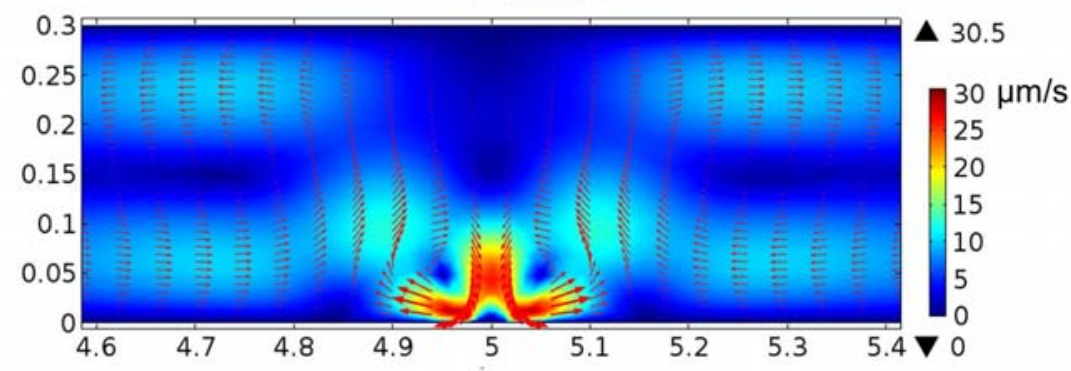

b

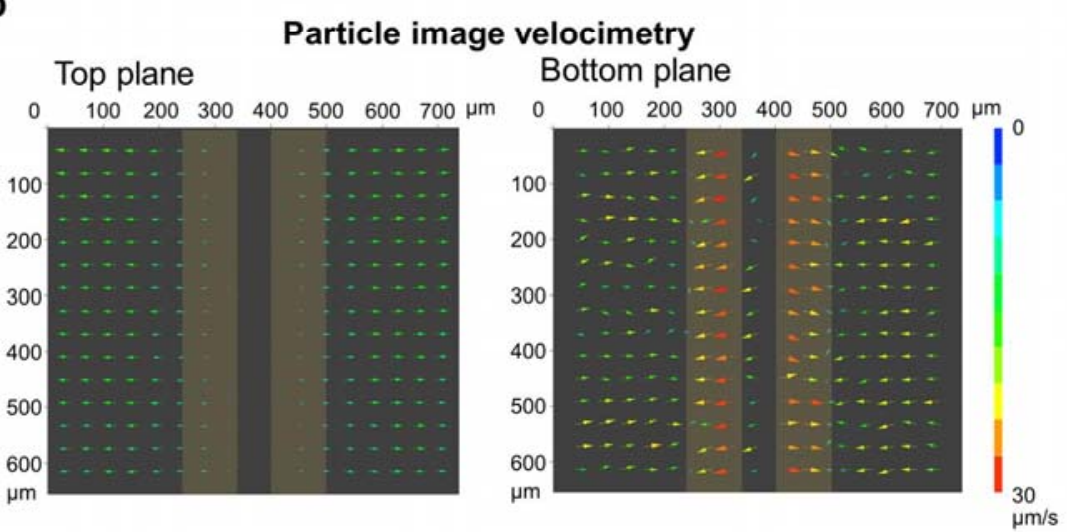

Figure 5. (a) The velocity profile in the $300 \mu \mathrm{m}$ height channel calculated using the extended model. (b) Particle image velocimetry measurement of the velocity profiles at the top and bottom plane of the microchannel.

\section{Regulation of the long-range fluid motion}

We further investigated the factors that regulate the long-range fluid motion using the extended computational model and experimental measurements. The voltage dependencies of long-range fluid motion in an $800 \mu \mathrm{m}$ channel were calculated in four different scenarios: the classical model, classical model with buoyancy, classical model with buoyancy and temperature dependent parameters, and the extended model (Fig. 6a). In the extended model, the buoyancy, the temperature-dependent parameters, and the third type boundary condition 
were incorporated. The velocity was measured in a region far from the electrode (Fig. 1). The velocity calculated in the classical model of ACEF is significantly lower that the measured values, showing the inability of the classical model in describing the long-range fluid motion. By incorporating buoyancy, the model resulted in reasonable agreement with the data up to 4 V. However, the model underestimated the velocity at higher voltage, which suggests that additional factors affected the long-range fluid motion. We therefore incorporated temperature dependent parameters in the model to capture the influence of the large temperature rise, which reduced the discrepancy between the model and the data. The model was further modified by using the third type boundary condition, resulting in the extended computational model of ACEF. We observed excellent agreement between the experimental data and the extended models $\left(R^{2}>0.99\right)$. The extended model also captures the fluid velocity in channels with different heights (Fig. 6b).
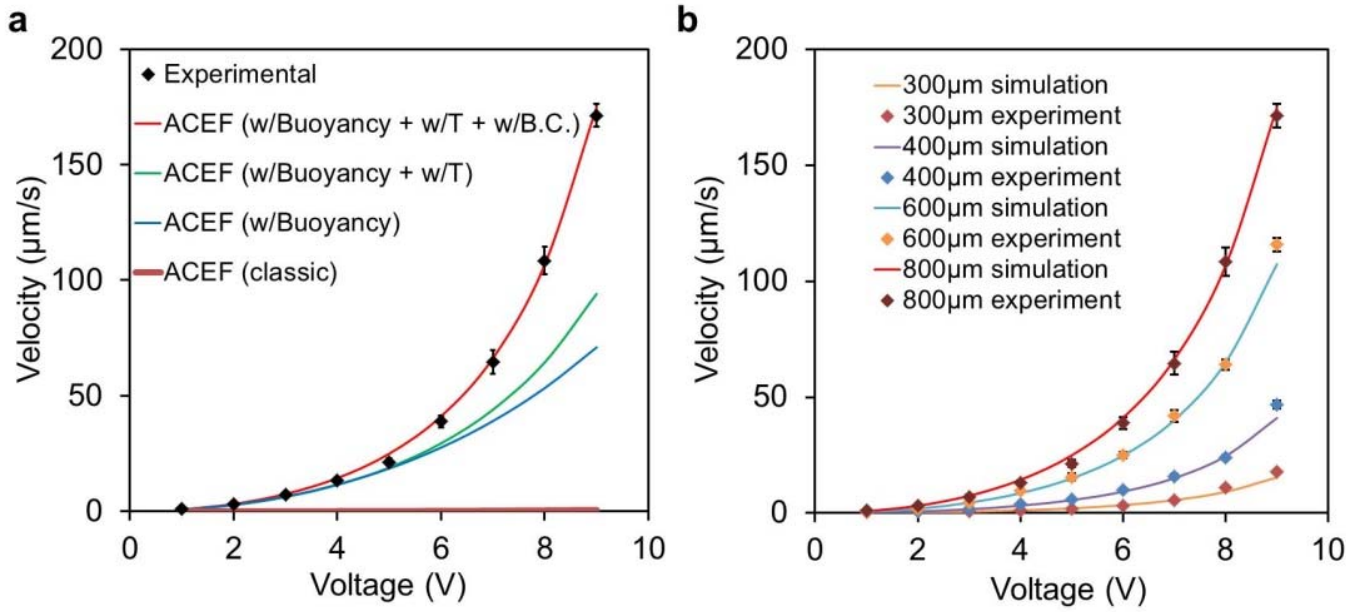

Figure 6 . The velocity profiles measured by particle image velocimetry (experimental) and calculated using the classical model of ACEF (classic), classical model with buoyancy (w/Buoyancy), classical model with buoyancy and temperature dependent parameters ( $w /$ Buoyancy $+w / T)$, and the extended model with buoyancy, temperature parameters and third type boundary condition 
(w/buoyancy+w/T+w/B.C.). The coefficient of determination were calculated respectively, $R^{2}=0.70719$ (for ACEF w/Buoyancy), $R^{2}=0.822703$ (for ACEF w/Buoyancy $+w / T$ ), and $R^{2}=0.999201$ (for ACEF $w /$ Buoyancy $+w / T+w / B . C)$. (b) The velocity profiles in channels with different heights. Data represent mean \pm standard deviation. The coefficient of determination of the fitting are $R^{2}=0.9662(300 \mu \mathrm{m})$, $0.9814(400 \mu \mathrm{m}), 0.9935(600 \mu \mathrm{m}), 0.9986(800 \mu \mathrm{m})$, respectively.

\section{DISCUSSION}

In this study, we identified a long-range fluid motion induced electrothermally. This centimeter scale fluid motion was at least 2 orders of magnitude larger than the previously observed ACEF fluid motion and could not be explained by the classical theory of ACEF. The fluid motion displayed strong dependencies on the applied voltage, sample electrical conductivity, and channel dimension. By developing an extended model of ACEF, we demonstrated that the buoyancy, the temperature-dependent parameters, and the third type boundary condition are critical factors for controlling this long-range fluid motion. The results were supported by the temperature and velocity profiles measured by infrared thermometry and particle image velocity. This long-range fluid motion was particular important for applications involving conductive solution (e.g., physiological fluid and biological buffers) and a large volume (large characteristic length of the system). For instance, a temperature rise of $10^{\circ} \mathrm{C}$ could increase the electrical conductivity for $20 \%$, resulting in non-linear increase in the temperature rise. The large temperature rise also leads to a large density gradient and buoyancy force. The relative significance of buoyancy and the onset of natural convection can be described by the Rayleigh number, Ra [25]. 


$$
\mathrm{Ra}=\frac{g \partial \rho / \partial T}{v k} \Delta T L^{3}
$$

where $v$ is the kinematic viscosity, $k$ is the thermal diffusivity, and $L$ is the characteristic length of the system. The Rayleigh number is highly sensitivity to the temperature rise and the length scale of the system. In the experiment, we observed a transition length scale of between 200 $\mu \mathrm{m}$ and $300 \mu \mathrm{m}$ with $7 \mathrm{Vpp}$. In the simulation, the maximum buoyancy induced velocity for length scale of 100,200 , and $300 \mu \mathrm{m}$ is $\sim 0.45, \sim 2.4$, and $\sim 6.8 \mu \mathrm{m} / \mathrm{s}$ respectively. As a comparison, the velocity associated with Brownian motion of the tracer particle is $\sim 2.5 \mu \mathrm{m} / \mathrm{s}$, supporting the transition length scale of the long range fluid motion.

Our study also revealed several critical issues in computational modeling of ACEF. First, the Dirichlet or fixed boundary condition is often used in numerical studies of ACEF. However, the fixed boundary condition is only valid for systems with a small temperature rise as verified by infrared thermometry. In our experiment, both the temperature gradient and velocity profile strongly depend on the appropriate boundary condition. The influence is of particular significance for conductive solutions with a high temperature rise. Second, several aspects of the electrical connection can often be overlooked in the experiment. For instance, one of the electrodes is typically grounded and square waves are often applied in the experiment. These factor should be carefully implemented in the simulation. Furthermore, the electrical potential across the electrode should be measured experimentally to minimize the error due to the finite resistance of the electrical wires. This is importance for manipulating biological fluids with high electrical conductivities.

Within the context of biomedical applications, the ability to create the long-range fluid motion may have important implications in the design of ACEF-based analytical devices. The 
miniaturization of diagnostic test platforms offers many advantages over centralized laboratory testing, such as reduced reagent volumes, rapid analysis, small size, low power consumption, parallel analysis, and functional integration of multiple devices. However, microfluidic systems are often limited by the volume mismatch between the microfluidics and the sample size in

practical applications. It is a fundamental obstacle that is necessary to address in order to move the technology toward clinical applications. The long-range fluid motion may enable novel techniques for manipulating the sample. Hybrid electrokinetics, i.e., combinations of different electrokinetic phenomena, can also be implemented to take advantages of electrokinetic techniques that are effective in different length scales.

\section{ACKNOWLEDGEMENTS}

This work was supported in part by the National Institutes of Health (R44AI088756 and DP2OD007161). The authors would like to thank Jose Miguel Valdez and Minqing Li for their valuable discussion and suggestions. 
Table 1

Physical properties and operation parameters

\begin{tabular}{|c|c|c|c|}
\hline Symbol & Value & Units & Notes \\
\hline$\sigma$ & $0.02425^{*}(T-273.15)+0.6883$ & {$[\mathrm{~S} / \mathrm{m}]$} & Electrical conductivity $(0.1 \mathrm{~m}$ \\
\hline & & & $\mathrm{KCL})$ \\
\hline$\varepsilon$ & $5321 \mathrm{~T}^{-1}+233.76-0.9297 \mathrm{~T}+0.1417 \times 10^{-2} \mathrm{~T}^{2}-0.829 \times 10^{-6} \mathrm{~T}^{3}$ & [Farad/m] & Relative permittivity (water) \\
\hline$k$ & $-0.869+0.00895 \mathrm{~T}-1.5837 \times 10^{-5} \mathrm{~T}^{2}+7.975 \times 10^{-9} \mathrm{~T}^{3}$ & {$[\mathrm{~W} /(\mathrm{m} \cdot \mathrm{K})]$} & $\begin{array}{l}\text { Thermal conductivity } \\
\text { (water) }\end{array}$ \\
\hline$\omega$ & $1,000,000$ & {$[\mathrm{~Hz}]$} & Frequency \\
\hline$g$ & 9.832 & {$\left[\mathrm{~m} / \mathrm{s}^{2}\right]$} & Gravitational acceleration \\
\hline$\rho_{m}$ & $838.466135+1.4005 \mathrm{~T}-0.00301 \mathrm{~T}^{2}+3.71822313 \mathrm{E}-7^{*} \mathrm{~T}^{3}$ & {$\left[\mathrm{~kg} / \mathrm{m}^{3}\right]$} & Density (water) \\
\hline$C$ & $12010.1471-80.4 \mathrm{~T}+0.31 \mathrm{~T}^{2}-5.38 \times 10^{-4} \mathrm{~T}^{3}+3.625 \times 10^{-7} \mathrm{~T}^{4}$ & {$[\mathrm{~J} /(\mathrm{kg} \cdot \mathrm{K})]$} & $\begin{array}{l}\text { Specific heat capacity } \\
\text { (water) }\end{array}$ \\
\hline$\mu$ & $\begin{array}{c}1.38-0.02 T+1.36 \times 10^{-4} \mathrm{~T}^{2}-4.65 \times 10^{-7} \mathrm{~T}^{3}+8.9 \times 10^{-10 *} \mathrm{~T}^{4}- \\
9.08 \times 10^{-13} \mathrm{~T}^{5}+3.85 \times 10^{-16} \mathrm{~T}^{6}\end{array}$ & {$[\mathrm{~Pa} \cdot \mathrm{s}]$} & Dynamic viscosity (water) \\
\hline
\end{tabular}




\section{REFERENCE}

[1] Y. Lu, T. Liu, A.C. Lamanda, M.L. Sin, V. Gau, J.C. Liao, P.K. Wong, AC Electrokinetics of Physiological Fluids for Biomedical Applications, J Lab Autom, (2014).

[2] M.L. Sin, K.E. Mach, P.K. Wong, J.C. Liao, Advances and challenges in biosensor-based diagnosis of infectious diseases, Expert Rev Mol Diagn, 14(2) (2014) 225-244.

[3] A. Ramos, H. Morgan, N.G. Green, A. Castellanos, Ac electrokinetics: a review of forces in microelectrode structures, Journal of Physics D-Applied Physics, 31(18) (1998) 2338-2353. [4] M.L. Sin, V. Gau, J.C. Liao, P.K. Wong, A Universal Electrode Approach for Automated Electrochemical Molecular Analyses, J Microelectromech Syst, 22(5) (2013) 1126-1132. [5] Y. Lu, J. Gao, D.D. Zhang, V. Gau, J.C. Liao, P.K. Wong, Single Cell Antimicrobial Susceptibility Testing by Confined Microchannels and Electrokinetic Loading, Analytical chemistry, (2013).

[6] M.L.Y. Sin, T.T. Liu, J.D. Pyne, V. Gau, J.C. Liao, P.K. Wong, In Situ Electrokinetic Enhancement for Self-Assembled-Monolayer-Based Electrochemical Biosensing, Anal Chem, 84(6) (2012) 2702-2707.

[7] J. Gao, R. Riahi, M.L. Sin, S. Zhang, P.K. Wong, Electrokinetic focusing and separation of mammalian cells in conductive biological fluids, Analyst, 137(22) (2012) 5215-5221.

[8] J. Gao, M.L.Y. Sin, T. Liu, V. Gau, J.C. Liao, P.K. Wong, Hybrid Electrokinetic Manipulation in High-Conductivity Media, Lab Chip, 11 (2011) 1770-1775.

[9] M.L.Y. Sin, V. Gau, J.C. Liao, P.K. Wong, Electrothermal Fluid Manipulation of HighConductivity Samples for Laboratory Automation Applications, Journal of Association for Laboratory Automation, 15 (2010) 426-432. 
[10] T. Liu, M.L. Sin, J.D. Pyne, V. Gau, J.C. Liao, P.K. Wong, Electrokinetic stringency control in self-assembled monolayer-based biosensors for multiplex urinary tract infection diagnosis, Nanomedicine, 10(1) (2014) 159-166.

[11] T. Liu, Y. Lu, V. Gau, J.C. Liao, P.K. Wong, Rapid antimicrobial susceptibility testing with electrokinetics enhanced biosensors for diagnosis of acute bacterial infections, Ann Biomed Eng, 42(11) (2014) 2314-2321.

[12] Z. Gagnon, J. Mazur, H.C. Chang, Integrated AC electrokinetic cell separation in a closedloop device, Lab on a Chip, 10(6) (2010) 718-726.

[13] E. Du, S. Manoochehri, Microfluidic pumping optimization in microgrooved channels with ac electrothermal actuations, Applied Physics Letters, 96(3) (2010) -.

[14] S. Park, M. Koklu, A. Beskok, Particle Trapping in High-Conductivity Media with Electrothermally Enhanced Negative Dielectrophoresis, Analytical chemistry, 81(6) (2009) 2303-2310.

[15] J. Oh, R. Hart, J. Capurro, H. Noh, Comprehensive analysis of particle motion under nonuniform AC electric fields in a microchannel, Lab on a Chip, 9(1) (2009) 62-78.

[16] W.Y. Ng, S. Goh, Y.C. Lam, C. Yang, I. Rodriguez, DC-biased AC-electroosmotic and ACelectrothermal flow mixing in microchannels, Lab Chip, 9(6) (2009) 802-809.

[17] H.C. Feldman, M. Sigurdson, C.D. Meinhart, AC electrothermal enhancement of heterogeneous assays in microfluidics, Lab Chip, 7(11) (2007) 1553-1559.

[18] M. Sigurdson, D.Z. Wang, C.D. Meinhart, Electrothermal stirring for heterogeneous immunoassays, Lab on a Chip, 5(12) (2005) 1366-1373.

[19] S. Loire, P. Kauffmann, I. Mezic, C.D. Meinhart, A theoretical and experimental study of ac electrothermal flows, J Phys D Appl Phys, 45(18) (2012). 
[20] F.J. Hong, J. Cao, P. Cheng, A parametric study of AC electrothermal flow in microchannels with asymmetrical interdigitated electrodes, Int Commun Heat Mass, 38(3) (2011) 275-279.

[21] C.D. Meinhart, S.T. Wereley, J.G. Santiago, PIV measurements of a microchannel flow, Experiments in Fluids, 27(5) (1999) 414-419.

[22] A.K. Prasad, R.J. Adrian, C.C. Landreth, P.W. Offutt, Effect of Resolution on the Speed and Accuracy of Particle Image Velocimetry Interrogation, Exp Fluids, 13(2-3) (1992) 105-116. [23] P.K. Wong, C.Y. Chen, T.H. Wang, C.M. Ho, Electrokinetic bioprocessor for concentrating cells and molecules, Analytical chemistry, 76(23) (2004) 6908-6914.

[24] C. Meinhart, D.Z. Wang, K. Turner, Measurement of AC electrokinetic flows, Biomedical microdevices, 5(2) (2003) 139-145.

[25] A. Castellanos, A. Ramos, A. Gonzalez, N.G. Green, H. Morgan, Electrohydrodynamics and dielectrophoresis in microsystems: scaling laws, Journal of Physics D-Applied Physics, $36(20)(2003)$ 2584-2597. 
